\title{
A Rare Tumor in the Common Bile Duct: A Case Report
}

\author{
Mustafa Suker, Katharina Biermann, ${ }^{2 *}$ Casper van Eijck, and Michael Doukas²
}

\begin{abstract}
Background: Lymphoepithelial-like carcinoma (LEC) is rarely found in organs outside the nasopharyngeal area. This is the first case report of Epstein-Barr virus (EBV)-associated LEC of the extrahepatic tract. As it is very difficult to distinguish between LEC and adenocarcinoma in the clinical presentation, this article can give more insight into how the pathological analysis can help with the diagnosis.

Case presentation: A 37-year-old Caucasian male with a history of Crohn's disease and primary sclerosing cholangitis presented with cholestasis. A computed tomography scan revealed a tumor in the pancreatic head without invasion into the surrounding organs. The patient underwent an uncomplicated pyloruspreserving pancreaticoduodenectomy, with pathology revealing an epithelial carcinoma of the common bile duct with metastases in 4 of the 18 resected lymph nodes. In situ hybridization demonstrated extensive EBV positivity in the tumor cells, and in serum, positive lgG anti-EBV was found. The diagnosis of EBV-associated LEC was hereby confirmed. The postoperative course was uneventful and 18 months after surgery there is no recurrence.

Conclusion: In the case of an epithelial tumor in the periampullary region, one should consider EBV-associated LEC as this tumor may have a lot of similarity with the adenocarcinoma but has lower rates of recurrence after surgery and better overall survival.
\end{abstract}

Keywords: biliary tract neoplasms; Epstein-Barr virus infections; lymphoepithelial-like carcinoma

\section{Introduction and Background}

Lymphoepithelial-like carcinoma (LEC) is usually seen in nasopharyngeal carcinoma and mostly associated with Epstein-Barr virus (EBV) infection. ${ }^{1}$ However, case reports have described the occurrence of LEC in the lung, salivary gland, trachea, thymus, esophagus, urinary bladder, uterine cervix, vagina, breast, renal pelvis, stomach, and the intrahepatic biliary tract. The diagnosis of biliary tract LEC is of importance to the physician as well to the patient, as these tumors are characterized by lower rates of recurrence and better overall survival. ${ }^{2}$ Here, we report the first EBV-related LEC of the extrahepatic biliary tract.

\section{Case Presentation}

A 37-year-old Caucasian male with a history of Crohn's disease and primary sclerosing cholangitis presented with cholestasis. A magnetic resonance cholangiopancreatography showed a tumorous process in the pancreatic head with a double duct sign. Endoscopic ultrasound revealed a hypoechoic lesion of $3 \mathrm{~cm}$ in the pancreatic head with a pancreatic duct dilatation of $5.5 \mathrm{~mm}$. Endoscopic retrograde cholangiopancreatogram was performed with placement of a pigtail stent in the pancreatic duct, but cannulation of the common bile duct was not possible. A computed tomography scan revealed a tumor in the pancreatic head without invasion into the surrounding organs (Fig. 1).

Departments of ${ }^{1}$ Surgery and ${ }^{2}$ Pathology, Erasmus MC, University Medical Center Rotterdam, Rotterdam, The Netherlands.

*Address correspondence to: Katharina Biermann, MD, PhD, Department of Pathology, Erasmus MC, University Medical Center Rotterdam, 's-Gravendijkwal 230, 3015 CE Rotterdam, The Netherlands, E-mail: k.biermann@erasmusmc.nl

(c) Mustafa Suker et al. 2017; Published by Mary Ann Liebert, Inc. This Open Access article is distributed under the terms of the Creative Commons License (http://creativecommons.org/licenses/by/4.0), which permits unrestricted use, distribution, and reproduction in any medium, provided the original work is properly credited. 


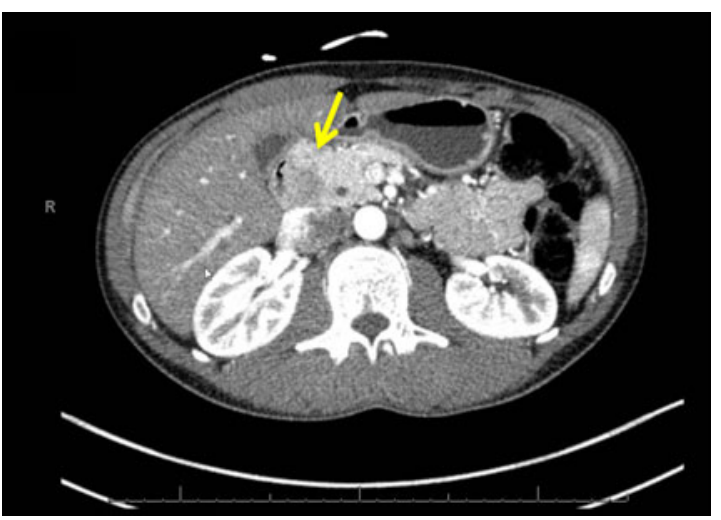

FIG. 1. Computed tomography scan with the yellow arrow pointing at the tumor.

As our working diagnosis was pancreatic cancer, our patient underwent an uncomplicated pyloruspreserving pancreaticoduodenectomy. The surgical specimen revealed a tumor arising in the common bile duct with pancreatic involvement, and possible extension to the duodenum and into surrounding fat tissue (Fig. 2). The histopathological sections showed poorly differentiated carcinoma of the common bile duct mostly with syncytial growth and prominent lymphoplasmacellular inflammatory component, consistent with LEC. Immunohistochemistry confirmed epithelial origin, since all tumor cells were positive for keratins (pankeratin and CAM5.2) and keratin19. In situ hybridization demonstrated extensive EBV

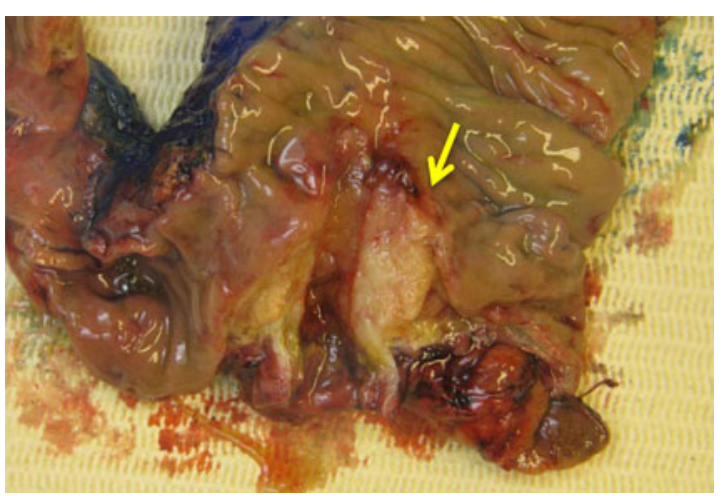

FIG. 2. Resection specimen with the yellow arrow pointing to the tumor in the common bile duct.

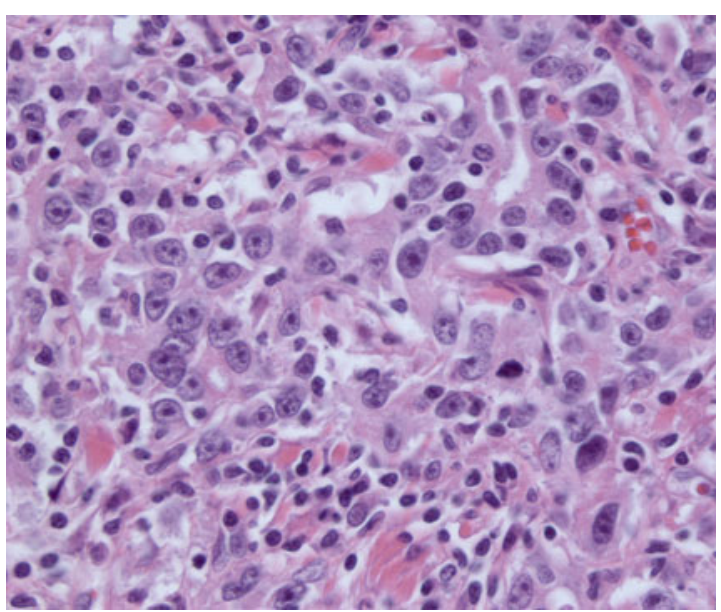

FIG. 3. Poorly differentiated carcinoma of the common bile duct on hematoxylin and eosin stain $\times 400$.

positivity in the tumor cells (Fig. 3: hematoxylin and eosin stain $\times 400$ and Fig. 4: EBV-encoded RNA stain $\times$ 400). Eighteen specimen lymph nodes were identified and four lymph nodes revealed carcinoma metastases with histopathological features of the primary. In the serum, IgG anti-EBV was positive while IgM antiEBV was negative. The diagnosis of EBV-related LEC was confirmed by professor R.H. Hruban (Johns Hopkins Hospital, Baltimore, USA). Postoperative course

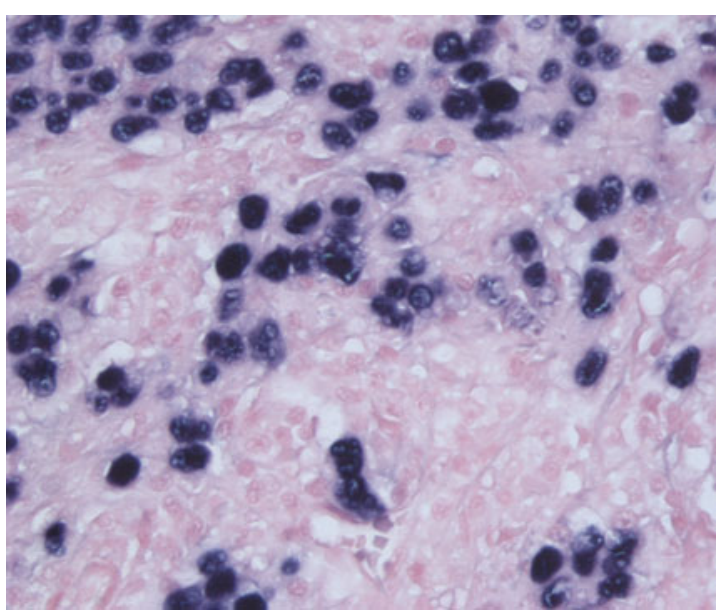

FIG. 4. In situ hybridization demonstrating extensive EBV positivity in the tumor cells on EBVencoded RNA stain $\times 400$. EBV, Epstein-Barr virus. 
was uneventful for our patient and 18 months after surgery there is no carcinoma recurrence.

\section{Conclusion}

EBV-associated LEC is rarely found in organs outside the nasopharyngeal area. Case reports described the occurrence of LEC in virtually every organ. ${ }^{2}$ To the best of our knowledge, there is only one published case that reported LEC in the distal bile duct, ${ }^{3}$ our case being the first EBVrelated carcinoma of the extrahepatic biliary tract.

\section{Acknowledgment}

We want to thank professor R.H. Hruban (Johns Hopkins Hospital, Baltimore, USA) for his expert opinion in this case.

\section{Authors' Contributions}

M.S. gathered clinical information and wrote the article, M.D. and K.B. performed the pathological analysis and revised and supervised the article, and C.v.E. provided the clinical information and revised the article.

\section{Author Disclosure Statement}

No competing financial interests exist.

\section{References}

1. Thompson LD. Update on nasopharyngeal carcinoma. Head Neck Pathol. 2007:1:81-86.

2. Solinas A, Calvisi DF. Lessons from rare tumors: Hepatic lymphoepithelioma-like carcinomas. World J Gastroenterol. 2015;21:34723479.

3. Ishida M, Mori T, Shiomi H, et al. Non-epstein-barr virus associated lymphoepithelioma-like carcinoma of the inferior common bile duct World J Gastrointest Oncol. 2011;3:111-115.

Cite this article as: Suker M, Biermann K, van Eijck C, Doukas M (2017) A rare tumor in the common bile duct: A case report, Journal of Pancreatic Cancer 3:1, 10-12, DOI: 10.1089/pancan.2016.0020.

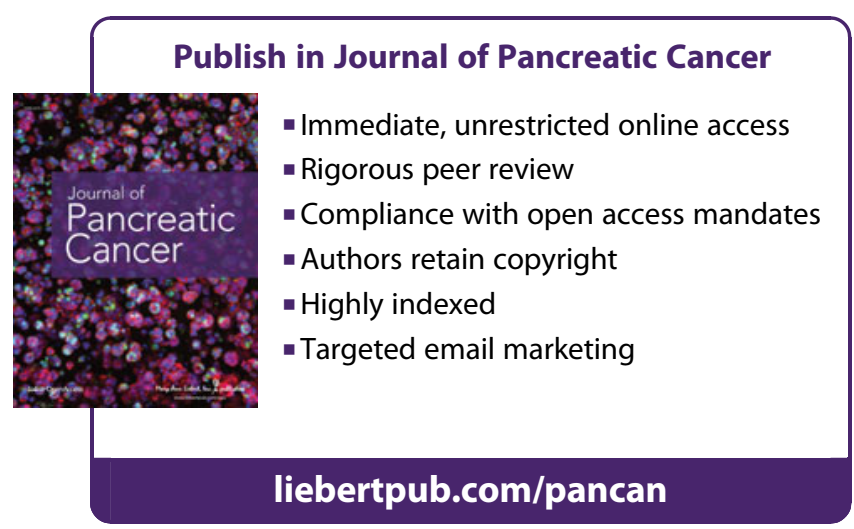

